Uršula Krevs Birk*

Domen Krištofelc**

Universität Ljubljana
UDK 811.163.6'28(497.452):81'373.45=112.2

DOI: $10.4312 /$ linguistica.60.2.193-210

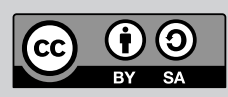

\title{
KONTAKT- UND VARIETÄTENLINGUISTISCHE ASPEKTE DES DEUTSCHEN LEHNGUTES IN DER MUNDART VON KROPA
}

\section{EINFÜHRUNG}

Der historische deutsch-slowenische Sprachkontakt ist durch die Tatsache gekennzeichnet, dass die Nachbarsprachen Deutsch und Slowenisch bis zum Ende des Ersten Weltkrieges koexistente Sprachen im slowenischen ethnischen Gebiet waren, und zwar nicht nur wegen deutschsprachiger Herrscher, die seit dem 8. Jahrhundert im slowenischen Raum deutsch und somit deutschsprachig waren, sondern auch durch zu verschiedenen Zeitperioden zugezogene Kleriker, Kolonisten, Facharbeiter, Beamte u. a., die aus vielen deutschsprachigen Regionen in verschiedenen slowenischen Gegenden angesiedelt wurden. In Koexistenz mit slowenischen Muttersprachlern trugen sie zur geschichtlichen Entwicklung der Orte oder Teile von Regionen bei, in denen sie entweder als (Sprach-) Minderheiten oder Mehrheiten oder sogar als (monolinguale) Sprachinseln existierten. Aus kontaktlinguistischer Sicht waren diverse Varietäten des Deutschen und Slowenischen an Sprachkontaktprozessen beteiligt, in denen sich die Sprachen gegenseitig beeinflussten (vgl. Krevs Birk 2019), bei der Einflussnahme hinterließ das Deutsche in der Regel viel stärkere Spuren in slowenischen Kontaktvarietäten als umgekehrt. Evidente Spuren der Einwirkung des Deutschen als Geber- oder Vermittlersprache sind diverse Arten von Entlehnungen, Phraseologismen, Toponymika, Numeralia, Interferenzerscheinungen und Kommunikationsformeln, durch die sich die deutsche und die slowenische Sprache sowohl als zwei typologisch weniger ähnliche als auch genetisch weit entfernte Sprachen eines (gemeinsamen) Sprachbundes (und auch Sprechbundes) in einem Kontaktareal festlegen lassen. ${ }^{1}$

Im vorliegenden Beitrag soll anhand der deutschen Lehnwörter in der Mundart von Kropa exemplarisch gezeigt werden, wie stark die deutschsprachigen Einwirkungen auf das Slowenische und seine Varietäten in der jahrhundertelangen

Ursula.KrevsBirk@ff.uni-lj.si

** domen.kristofelc@gmail.com

1 Ein Sprachbund besteht (im Sinne von Trubetzkoy) aus Sprachen, die im Laufe der Geschichte in einem bestimmten geographischen Gebiet durch Interferenz gemeinsame strukturelle Eigenschaften ausbilden. Glovacki-Bernardi (2011: 176) definiert auch den Begriff Sprechbund, der aus Sprachen besteht, die Ähnlichkeiten in der Realisation von pragmatischen Kommunikationsmustern aufweisen. Beide Begriffe korrelieren mit dem Begriff Sprachareal (vgl. Newerkla 2017: 21). 
Sprachkontaktgeschichte waren, obwohl der Sprachkontakt, der Anfang des 20. Jahrhunderts durch die (noch) Verwaltung Österreich-Ungarns gesichert war, durch den I. Weltkrieg abgebrochen wurde. Solch ein reges Sprachkontaktgeschehen wickelte sich bis dahin auch in der Geschichte des Ortes Kropa ab. Die heute relativ kleine Ortschaft Kropa, eine Marktsiedlung in der Region Gorenjsko (Oberkrain) im nordwestlichen Teil Sloweniens, die auch weit über die slowenischen Grenzen hinaus für ihre ehemalige Eisenverarbeitung bekannt war - ihr Ortsname war im 19. Jahrhundert noch zweisprachig (dt. Bergwerk Kropp - slow. Fužina Kropa, Škofic 2019: 17)² -, war nämlich in der Geschichte stark mit den deutschen Muttersprachlern verbunden, die als Kolonisten aus österreichischen, vorwiegend Kärntner Regionen im 14. Jahrhundert im Kropa-Tal angesiedelt wurden. Die Interaktion zwischen den deutschen und slowenischen Muttersprachlern im Laufe mehrerer Jahrhunderte hinterließ in der Sprachvarietät der Ortschaft evidente Spuren, denn zum festen Bestandteil ihres Wortschatzes gehören zahlreiche deutsche Lehnwörter bzw. lexikalische Germanismen, die u. a. die dialektal stark ausdifferenzierte Mundart von Kropa kennzeichnen. Der Beitrag setzt sich zum Ziel, nach der Darstellung der geschichtlichen Entwicklung der Ortschaft im Lichte der wirtschaftlichen und soziokulturellen Kontakte mit dem deutschsprachigen Raum den deutsch-slowenischen Sprachkontakt in Kropa exemplarisch anhand der umgangssprachlichen bzw. mundartlichen lexikalischen Germanismen darzustellen. Neben der Festlegung der deutschen Geber-Dialekte wie auch der Kommunikationsbereiche, in denen sie vorkommen, wird versucht zu zeigen, inwiefern sie in den aktuellen slowenischen Varietäten, wie etwa überregionalen oder spezifisch mundartlichen, verankert sind. Darüber hinaus wird auch der Frage nachgegangen, ob sich - in einem weiteren Varietätenraum zweier oder auch mehrerer Kontaktsprachen, wie etwa des Slowenischen und Kroatischen - eine Konvergenz der entlehnten Lexika abzeichnet, die ein gemeinsames Kontaktareal mit deutscher Verkehrssprache österreichischer Prägung ausweist. ${ }^{3}$

\section{GESCHICHTE DER DEUTSCH-SLOWENISCHEN KONTAKTE IN KROPA UND UMGEBUNG}

Seit Ende des 12. Jahrhunderts gehörte das Gebiet, das sich heute in der Gemeinde Radovljica befindet, zur Grafschaft Ortenburg. Bekanntlich siedelten die Ortenburger die Kolonisten in unbesiedelten Regionen an, die sie in Besitz hatten. Die größte solcher Regionen im slowenischen Gebiet lag in der Unterkrain - die Gottschee, in die die Bauern und ihre Familien im 14. Jahrhundert geschickt wurden und mit der Zeit im kargen Karstland eine homogene deutsche Sprachinsel bildeten.

In der Oberkrain wurden die Kolonisten im Kropa-Tal angesiedelt, was stark mit den Erzlagerstätten um die Karst-Hochebene Jelovica und der Eisenverarbeitung in

2 Jožica Škofic, die zur Mundart von Kropa forscht und sich auch mit der Mikrotoponomastik des Ortes beschäftigt, hält fest, dass der Siedlungsname Kropa vom Appellativum kropa kommt (2019: 15), was ,starke Wasserquelle“ bedeutet und aus *kropa entstand, das eigentlich ein Femininum aus einem substantivierten Adjektiv im Slawischen ist (Škofic 2019: 15, nach Snoj 2009).

3 Zum Kontaktareal s. Anm. 2. Vgl. Krevs Birk (2019: 166-167). 
den Orten unter der Hochebene zusammenhängt. ${ }^{4}$ Nach einigen Angaben besiedelten Nagelschmiede aus Ferlach schon vor dem 14. Jahrhundert die Gebiete unterhalb der Alm Korošica. ${ }^{5}$ Das Kärntner Eisenhüttenwesen hatte damals eine eher lange Tradition, da die Kärntner schon früher Nägel für den Mittelmeerraum produziert hatten. Die Zuwanderer aus Kärnten, besonders aus Ferlach, waren Facharbeiter mit ihren Familien, deren Facharbeit auf denselben Techniken der Eisenverarbeitung wie in Kärnten basierte. Die Niederlassung in Kropa kam auch Händlern aus Venedig zugute, denn der Weg zu ihren Geschäftspartnern im Süden wurde einfacher und kürzer, da sie nicht mehr über den Loiblpass nach Kärnten reisen mussten. Da es in Kropa damals schon zwei Hammer- und mehrere Eisenwerke gab, war dieser Ort auch für die Arbeitskräfte aus den Nachbarorten interessant. Im Mittelalter wuchs die Nachfrage nach Eisenprodukten und es kam zur Entwicklung neuer Verarbeitungstechniken. Das führte zum Austausch von Ideen, Arbeitern und Technologien innerhalb und außerhalb der oben erwähnten Regionen. Der aufblühende Handel stärkte die Transportwege, die von Kärnten über den Loiblpass durch Neumärktl/Tržič, Radmannsdorf/Radovljica und Kropp/Kropa weiter nach Norditalien bis Venetien führten. Kropas Schmiede suchten Verkaufsmärkte im Süden und Südwesten, da die nördlichen Märkte in Kärnten und in der Steiermark von den Schmieden nördlich der Karawanken bedient wurden. Kropa wurde somit schon gegen Ende des Mittelalters zum Träger des Kultur- und Sprachkontaktes zwischen mehreren Sprachen (Slowenisch, Deutsch, Italienisch, Friaulisch, Kroatisch) und Sprachvarietäten (Mundarten aus der Krain und Kärnten).

Das Eisenhüttenwesen brachte die Ortschaft allmählich zum Aufblühen. Den Aufschwung der Ortschaft stärkte insbesondere auch die Bergbauordnung, die der Ortschaft 1550 von Kaiser Ferdinand I. verliehen wurde, wodurch sie eine weitgehende Autonomie erhielt. Danach wurden die Beziehungen zwischen den Grundbesitzern und den Schmieden geregelt, aber auch weitere Privilegien wurden ihnen gesichert: Die Ortsbewohner brauchten weder Steuern zu zahlen noch Militärdienst zu leisten, was über zweihundert Jahre, bis zur Reform Josephs I. galt. In der Blütezeit gab es dort mehr als 70 Häuser mit 1400 Einwohnern, die bis zu zwei Tonnen Eisen pro Jahr verarbeiteten.

Auch Valvasor hob die Bedeutung Kropas in seiner Die Ehre des Herzogtums Krain (1689) hervor, indem er das Bergwerk Hammersgewerg Cropp (1689, I./III.: 386-387) darstellte:

4 Nach Bogožalec (2001: 12) verbreiteten die Ortenburger die Eisenverhüttung im slowenischen Raum in Kropa durch die Ansiedlung der Kolonisten aus Kärnten und weiteren Regionen. Unter dem Grafen Friedrich II. von Ortenburg sollten auch Bergarbeiter aus Sachsen im Jahre 1349 in Kropa angesiedelt worden sein.

5 Der Überlieferung nach hatten sich die Nagelschmiede aus Ferlach allerdings schon im 14. Jahrhundert diesseits des Loiblpasses angesiedelt. Der Bergrutsch vertrieb sie ins Tal, wo die Ortschaft Neumarktl/Tržič entstand. Einige von ihnen wurden auf ihrem weiteren Weg in Kropa ansässig. Dafür spricht auch die Tatsache, dass in der Mundart von Ferlach und der Mundart von Kropa einige gemeinsame sprachliche Besonderheiten zu finden sind, wie etwa das R-Rollen (vgl. Gašperšič 1956: 11-12). Es besteht aber noch eine dritte Überlieferung, nach der die Einwohner von Kropa eigentlich Einwanderer aus Kärnten oder sogar aus Tirol waren (ebd.). 


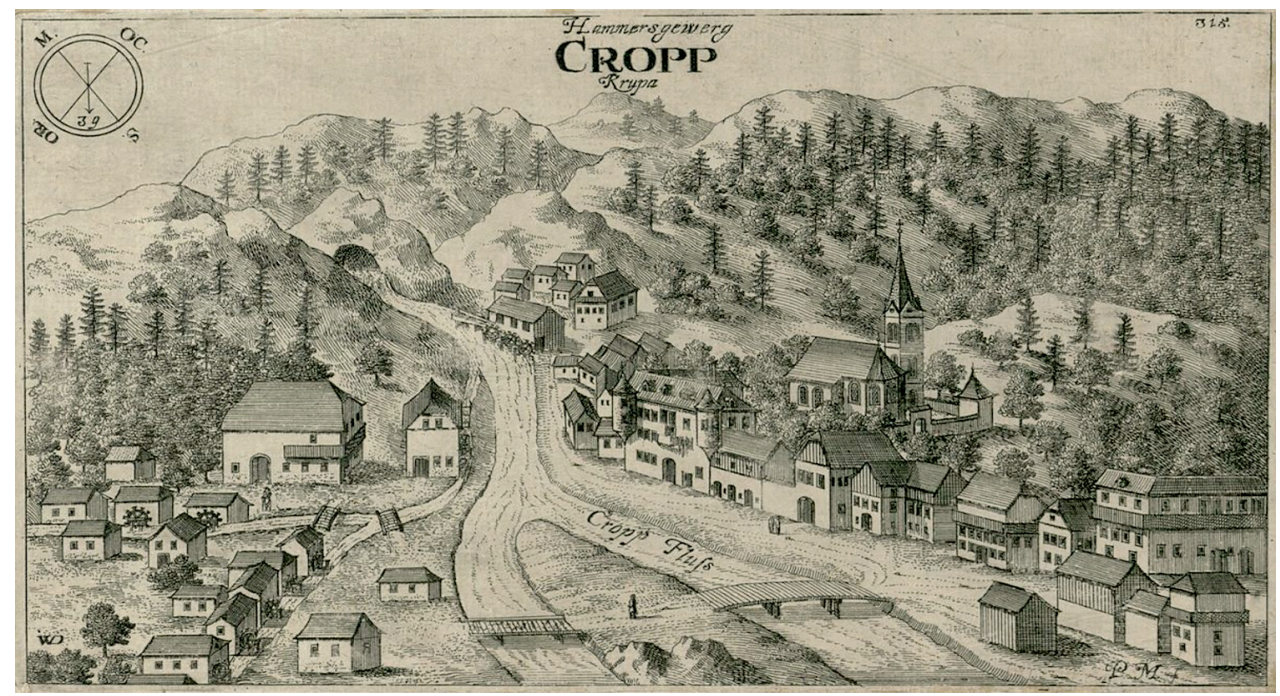

Abb. 1: Hammersgewerg Cropp (Die Ehre des Herzogtums Krain 1689, I./III., 386)

Auf der Illustration (Abb. 1) ist die „Gelegenheit oder Lager des Bergwercks Cropp“ abgebildet, darauf ist sehr deutlich das deutsche Toponym Cropp (mit dem Zusatz Hammerbergwerg) wie auch das slowenische Krupa zu lesen (sowie der Flussname Cropp, im Fließtext auch Cropa) (ebd.: 386). Valvasor beschreibt das Bergwerk wie folgt:

In Ober=Crain / ligt gleichfalls das Bergwerck Cropp / so man insgemein sonst Kropa nennet / in einer Schlutten oder Graben / zwischem hohem Gebirge / nicht fern von der Stadt Rattmannsdorff / und ist Lands=Fürstlich. Es wird ebenfalls / in zwei Theyle / nemlich in Ober- und Unter=Cropp / unterschieden: die aber nicht weit / sondern allein / biß auf eine Halbviertheil Stunde / voneinander entsessen seynd. Es ist mit wolgemaurten Häusern geziert / wie die ganz eigentlich darnach gerissene Figur zu erkennen giebt.

Daselbst entspringt die Cropa: welches Gewässer / aus dem Felsen eines Berges / als wie aus einem Kessel / hervorspringt.

Bey selbigem seinem Ursprunge besizt diß Wasser eine medicinirende Krafft: sintemal es / eingetrunken / laxirt und purgiret; also / daß ein Solcher / der einer Blut=Reinigung vonnöthen hat / sich wol darnach befindt.

Allhie wird das Werck / eben sowol / auf die Wölfe / gearbeitet: woraus man folgends allerley Nägel / Garter=Eisen / und dergleichen Sachen schmiedet. Es haben / vor einiger Zeit / die Nägel / so allhie gemacht werden / an ihrem Ruhm / etwas eingebüsst: Weil die Arbeiter damit nicht allerdings getreulich umgegangen / sondern sowol an der Zahl / als am Gewigt / denselben ein wenig abgebrochen: Aber solches ist nunmehr abgestellt / und die richtige Voll=Lieferung wieder eingeführt worden.

Es wohnen daselbst lauter Hammer $=$ Gewercke (oder Hammer $=$ Meister) $d a-$ runter / jetziger Zeit / die fürnehmsten seynd / deß Stephan Mazol sel. Erben / Sebastian Scheganz/ Jacob / und Gregor Gasperin. (ebd.: 386-387) 
Trotz der Anmerkung Valvasors, ,,an ihrem Ruhm etwas eingebüsst“" zu haben, galt Kropa zu dieser Zeit als ein bedeutender, für die Nägelproduktion wichtiger Ort in der Region. Dies änderte sich Ende des 18. Jahrhunderts, als die Nachfrage nach Nägeln nachließ, was die Bewohner in existenzielle Not brachte. Dies wiederholte sich auch im 19. Jahrhundert. Als die Napoleonischen Kriege zum Ende der Venetianischen Republik beitrugen und eine Krise in der Habsburgermonarchie verursachten, verloren die Kärntner Schmiede und dadurch auch die Schmiede aus Kropa ihren wichtigsten Markt. Der letzte Schlag für die Industrie in diesen Gebieten waren aber die Entwicklung der Eisenbahn und zugleich der Mangel an Eisen in Gebieten um die Jelovica, so dass die Eisenerzgewinnung 1880 eingestellt wurde.

Der Mangel an Eisenerz sowie die immer stärkere Eisenindustrie, mit welcher die Handwerker in Kropa nicht mehr mithalten konnten, verursachte auch Auswanderung. Viele Menschen waren gezwungen, Kropa zu verlassen. Um das Problem mit dem Eisenerzmangel zu lösen, kauften Schmiede aus Kropa das Eisen in den Zentren der Eisenverarbeitung in Kärnten, vor allem in Hüttenberg, während sie in Ferlach und Saaz die Eisenüberreste kauften, die bei der Nagelproduktion entstanden. In den achtziger Jahren des 19. Jahrhunderts waren die Kontakte zwischen Kropa und Kärnten so stark, dass die Kärntner Behörden, die die dortige Lage verbessern wollten, auch nach Lösungen für Kropa suchten. Eine der Ideen war z. B. die Zusammenarbeit mit der Waffenindustrie in Ferlach, da man dort aufgrund der steigenden Anfrage nicht alles selbst produzieren konnte. Kropa blieb aber noch weiter ein wichtiger Geschäftspartner der österreichischen Orte - in Klagenfurt kaufte man nämlich Holz, in Ferlach Eisen und in Villach Kohlenkoks. Auch zur Zeit des Ersten Weltkrieges kam die enge Verbundenheit zwischen Krain und Kärnten zum Ausdruck, da viele Soldaten aus Kropa in den Einheiten aus Kärnten dienten. Viele Bewohner von Kropa sind während der Krise dazu nach Kärnten umgezogen, da sie sich erhofften, von besseren wirtschaftlichen Bedingungen zu profitieren. Das rege Kontaktgeschehen zwischen Kropa und Kärnten wurde durch den Ersten Weltkrieg aufgehalten.

\section{DEUTSCHE ENTLEHNUNGEN IN DER MUNDART VON KROPA}

\section{1}

Historische Sprachkontakte des Deutschen und des Slowenischen hinterließen evidente Spuren in der Mundart von Kropa. Wie bekannt ist für fremdsprachige Einflüsse die lexikalische Ebene am Zugänglichsten, so dass in der Sprachvarietät von Kropa erwartungsgemäß viele Lehnwörter aus dem Deutschen präsent sind. Im Wortschatz finden sich aber auch Spuren weiterer Kontaktsprachen, wie etwa des Italienischen und des Friaulischen, aber auch der Sprachvarietäten des Slowenischen, wie etwa der Mundart aus dem benachbarten Selca-Tal (Selška dolina) und der Kärntner Mundarten. Die Germanismen sind ein fester Bestandteil des dialektalen Wortschatzes aus dieser Gegend und genießen sowohl unter den älteren als auch unter den jüngeren Bewohnern dieser Orte nach wie vor große Popularität. 


\section{2}

In der Vergangenheit war im Rahmen historio- und ethnographischer Arbeiten über Kropa meistens der spezielle Wortschatz, der durch die Eisenproduktion aus der Vergangenheit generiert wurde, dargestellt (Müllner 1909, Gašperšič 1956, Šmitek 1980 u. a.), und auch sonst wurde Kropa bislang unter mehreren, wie etwa musikologischen, archäologischen, geografischen und geologischen Aspekten erforscht. Zur Mundart von Kropa im weitesten Sinne, zur Toponomastik in Kropa wie auch zum Fachwortschatz aus Kropa forscht ausführlich die Dialektologin Jožica Škofic. ${ }^{6}$

\section{3}

Wie der traditionellen Dialektkarte für slowenische Dialekte (Zemljevid slovenskih narečij von Tine Logar) ${ }^{7}$ zu entnehmen ist, gehört die Mundart von Kropa zum Gorenjsko-Dialekt (gorenjsko narečje bzw. Oberkrainer Dialekt), der zu den Dialekten der Gorenjsko-Dialektgruppe (gorenjska narečna skupina) ${ }^{8}$ gehört. Auf der Dialektkarte (s. Abb 2) ist Kropa im nördlichen Teil Oberkrains angemerkt.

Die Mundart wurde von Škofic $(1997,2019)$ aus phonologischer und morphologischer Sicht akribisch beschrieben. Sie hebt sich von den benachbarten Mundartvarietäten der Gorenjsko-Dialekte deutlich ab, und zwar in phonologischer Hinsicht (wie etwa das System der langen Vokale, Aussprache von $-l$ am Wortauslaut statt - $u$, die Aussprache des uvularen R, slow. „pogrkovanje“ u. a. vgl. Škofic 2019). Die Aussprache des Rachen-R, die in Nachbarmundarten nicht vorhanden ist bzw. die die Kropa-Mundart unter den Gorenjsko-Dialekten ausdifferenziert, ähnelt derjenigen in den Kärntner Dialekten (wie etwa aus dem Rosental), was als Zeugnis des intensiven Kontaktgeschehens zwischen Kropa und Südkärnten verstanden wird. Die Einwohner von Kropa sagen über sich selbst, dass sie einen kärntnerischen Dialekt sprechen. Wie bereits erwähnt haben die Stahlarbeiter aus Kärnten, die wegen der Entwicklung der Eisenverarbeitung schon im 14. Jahrhundert nach Kropa umgezogen sind, einen großen Einfluss auf die Sprache dieses Ortes ausgeübt. Wie Škofic (2019: 305) hervorhebt, trug zur Ausdifferenzierung der Mundart von Kropa stark seine abgelegene, in der Natur keinesfalls aufgeschlossene geographische Lage des Ortes bei, in die in der Blütezeit der Eisenproduktion weniger Sprecher aus nahegelegenen Nachbarorten und mehr aus weiteren Regionen zuzogen (wie etwa Kärnten und Selca-Gebirge). Kropa war jahrhundertelang eher von Orten in dessen Nähe getrennt, da das Tal, in welchem es sich befindet,

6 In Škofic 2019 sind 35 Beiträge über die Kropa-Mundart und ihre dialektologische Einbettung aufgelistet.

7 Karta slovenskih narečij z večjimi naselji. https://fran.si/204/sla-slovenski-lingvisticni-atlas/ datoteke/SLA_Karta-narecij.pdf.

8 Das slowenische Dialektkontinuum ist auf einer relativ kleinen Fläche breit gefächert: Die 36 Grunddialekte (narečje) und 13 Subdialekte (podnarečje) gruppiert man in sieben BasisDialektgruppen (gorenjska, dolenjska, panonska, štajerska, koroška, primorska, rovtarska narečna skupina), vgl. die Karte der slowenischen Dialekte, https://fran.si/204/sla-slovenski-lingvisticniatlas/datoteke/SLA_Karta-narecij.pdf. 


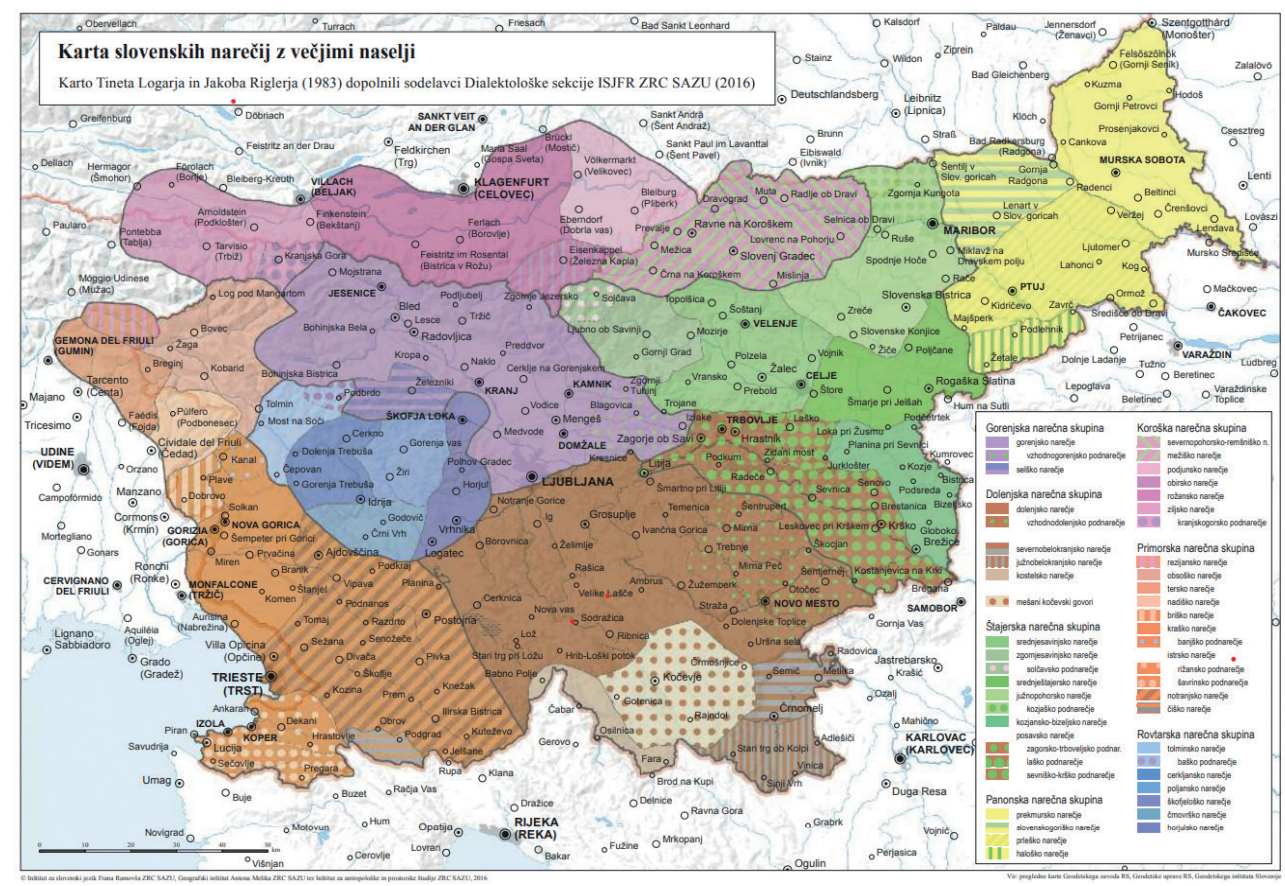

Abb. 2: Landkarte der slowenischen Dialekte mit größeren Siedlungen. Die Dialektkarte von Tine Logar und Jakob Rigler (1983) wurde von den Mitarbeitern der Dialektologischen Sektion des Fran-Ramovš-Instituts für slowenische Sprache des Forschungszentrums der Slowenischen Akademie der Wissenschaften und Künste ergänzt. Karta slovenskih narečij z večjimi naselji (2016). https://fran.si/204/sla-slovenski-lingvisticni-atlas/datoteke/SLA_Karta-narecij.pdf.

schwer zugänglich war. Seit der Ansiedlung lebten hier eher wenige Bauern (im Vergleich zu den Nachbardörfern). Škofic (ebd.) hebt des Weiteren als einen wichtigen Faktor der Ausdifferenzierung die relativ frühe Gründung der Pfarrgemeinde (1620) im Vergleich zur Nachbargemeinde Kamna Gorica (1751) hervor. In diesem Kontext war die soziale Homogenität der Bevölkerung entscheidend für die mundartspezifische Ausformung der Sprache, denn die Berufe und die Bevölkerung waren vorwiegend mit der Eisenproduktion verbunden, so dass sich die spezifischen, mit dem Eisenhüttenwesen verbundenen Begriffe etablieren konnten.

Der spezifische Fachwortschatz findet sich auch in der Mundart. Wie oben erwähnt spielten bei solcher Entwicklung auch die Germanismen eine sehr große Rolle, was besonders bei der Analyse der Namen für Schmiedewerkzeuge bzw. Schmiedegeräte evident wird. Die Bezeichnungen der Nägel ${ }^{9}$, die zum Teil der Standardsprache wurden, stammen hingegen nicht aus der deutschen, sondern vor allem aus der italienischen Sprache. Der Grund dafür liegt vor allem in dem marktorientierten

9 Einst wurden in Kropa über hundert Nägelsorten produziert, einige davon wurden beim Bau von Venedig verwendet. 
Schmiedehandwerk, denn fast alle Nägel wurden nach Italien geliefert. Einige umgangssprachliche Wörter deutscher Herkunft für Nägel bestehen aber nach wie vor, wie etwa jeger, das in der Sprachvarietät Kropas einen Nagel, der für die Bergschuhe geeignet war, bezeichnet. Das Lexem ist überregional ein Lehnwort mit derselben Bedeutung wie sein deutsches Herkunftswort (jager/jeger ,Jäger'), und in der sekundären Adaption entwickelte das Wort in der Kropa-Mundart eine spezifische Semantik (,Nagelsorte'). Aus dem Deutschen kommen auch das Wort šifnegel (,Nagel für den Schiffbau') oder maverhakel (,Mauerhaken'). Die Schmiede verwendeten bei ihrer Arbeit eher umgangssprachliche Begriffe, während die Standardbezeichnungen aus dem Deutschen wie auch aus dem Italienischen eher selten den Weg in die Alltagssprache der Handwerker fanden.

\section{4}

Die im Folgenden präsentierten deutschen Entlehnungen im Bereich der Lexik wurden den Kurzgeschichten Kroparske zgodbe („Geschichten aus Kropa“) entnommen, ${ }^{10}$ womit exemplarisch auf zahlreiche deutsche Entlehnungen in dieser Mundart hingewiesen werden soll. Der dialektologischen Studie von Škofic (2019) zu den Eigenschaften der Mundart auf der phonologischen, phonetischen und morphologischen Ebene ist zu entnehmen, dass auch Entlehnungen als ein unabdingbarer lexikalischer Bestandteil der Kropa-Mundart alle morphonologischen und phonetischen Feindifferenzen aufweisen, worauf im Rahmen dieses Beitrags nicht eingegangen wird.

Der Autor Jože Bertoncelj, Kunstschmiedemeister und Volkskundler aus Kropa, veröffentlichte im Jahr 197338 Kurzgeschichten, mit denen er ein stilistisch gelungenes literarisches Werk über das Leben in Kropa schuf. Er verfasste sie teilweise in der Kropa-Mundart, vor allem in den Dialogen, die dadurch authentisch erscheinen. Im Werk werden viele auf das Alltagsleben in Kropa in der Gegenwart und Vergangenheit bezogene Themen behandelt, die einen guten Überblick über die soziokulturelle Entwicklung der Ortschaft bieten. In der verschrifteten Mundart finden sich auch viele deutsche Lehnwörter, aber auch Lehnwörter aus dem Italienischen und Friaulischen. Dabei wurden die standardsprachlichen Lexeme, die deutscher Herkunft sind, nicht beachtet, da sie Teil des standardsprachlichen Bestandteils der slowenischen Lexik sind, für die in der Regel typisch ist, dass sie in einer sehr frühen Phase des Sprachkontaktes (s. Krevs Birk 2019: 56) aus dem Westgermanischen und Althochdeutschen ins damals dialektal sehr differenzierte Slowenisch kamen und vor der Standardisierung des Slowenischen (im 16. Jahrhundert) allgemein akzeptiert wurden. Wie Javor Briški (2020: 201) erklärt, kann man dies exemplarisch am heutigen slowenischen Wort skrinja (,Schrein') beobachten, das (monophtongiert) aus dem Althochdeutschen bzw. Mittelhochdeutschen entlehnt wurde (mhd. schrīn, ahd. skrini ,Gefäß‘, < lat. scrīnium). Angesichts des nhd. Schrein ist die deutschsprachige Herkunft beim Formativ skrinja nicht mehr evident, was ausschlaggebend bei der späteren

10 Die Erfassung der Germanismen basiert teilweise auf Recherchen, die im Rahmen der unter wissenschaftlicher Betreuung von Uršula Krevs Birk entstandenen Magisterarbeit von Domen Krištofelc (2019) durchgeführt wurden. 
sprachpuristischen Ausdifferenzierung und Ableitung der deutschen Lehnwörter, die formativisch ihren Herkunftswörtern ähnelten, in den slowenischen Substandard ${ }^{11}$ war. Solche Wörter in Kroparske zgodbe sind etwa šipa ,Scheibe', malica ,Mahlzeit‘ oder mežnarija ,Messnerei'.

Von Interesse für unsere Untersuchung sind vor allem deutsche Lehnwörter, die (noch) nicht in der slowenischen Standardsprache verankert sind.

Es wird festzustellen versucht, welche Kommunikationsbereiche diese Lexik widerspiegelt, aus welchen Chronolekten und Dialekten sie übernommen wurde, in welchen substandardsprachlichen Varietäten des Slowenischen diese Lexeme eventuell vorkommen und ob welche davon auch als konvergente Lehnwörter aus dem Deutschen in weiteren Kontakträumen, wie etwa mit der Kontaktsprache Kroatisch, erscheinen. Besonders interessant sind dabei Wörter, die man vielleicht heutzutage eher selten hört, die aber noch immer einen wesentlichen Teil der Lexik ausmachen.

In den mundartlich verschrifteten Teilen der Geschichten von Kropa wurden somit über hundert substandardsprachliche Lexeme gefunden, die aus der deutschen Sprache oder durch die deutsche Sprache in die Sprachvarietät Kropas gelangten. Bei den festgestellten Germanismen lassen sich folgende Kommunikationsbereiche erkennen:

- Mensch, Alltag, Kleidung, menschliche Kommunikation, wie etwa antret (,Antwort'), bogati (,gehorchen, folgen'), durhcug (,Durchzug'), gloncen (,polieren'), kešt (,Kost, Nahrung'), pogruntati (,ergründen, feststellen'), pubəč (,Bub'), regirati (,entscheiden') ${ }^{12},($ s)cagati (,zagen'), šansa (,Chance'), šmir (,Schmiere'), štibala (,Röhrenstiefel'), štupa (,Pulver'), varžet (,Hosentasche'), vestja (,Weste'), žajfa (,Seife'), župa (,Suppe'), drek/djek (,Dreck');

- menschliche Eigenschaften betreffend: fejst (,feist, fett'; ,stattlich'), fertik (,vollendet'), firbec (,e/r Schaulustige), flavzarija (,närrischer Einfall'), flavzati (,Flausen machen'), golant (,höflich'), kregan (,beschimpft'), kufrast (, wie aus Kupfer'), naštiman (< naštimati se; , aufgeputzt'), šentana (para) (,verfluchter Kerl'), vandrovec (,Wanderer');

- Haushalt, Zuhause: čevdr (,Keller'), kovtar (gesteppte) Bettdecke'), lampica (auch: lampa, einfache kleine Lampe'), pant (,Türband'), pisker (,Topf'), šajtrga (,Schubkarren'), štenga/štenža (,Stiege, Treppe'), štuk (,Stock, Etage $\left.{ }^{6}\right)^{13}$, boršt (,Forst, Wald');

- Wirtschaft, Geld: cvangarca (,Zwanziger'), činž (,Zins'), finfar (,Fünfer'), foršus (,Vorschuss'), kasa (,Kasse'), kontribucjon (,Kontribution'), kontroht (,Kontrakt, Vertrag'), kvit (,quitt'), likof (,Leikauf'), namalati (,aufschreiben'), odrajtati (,zahlen, abbezahlen'), ofer (,Kollekte'), priftošal (,Brieftasche'), rajtenga (,Rechnung, Lohn'), tavžent (, Tausend'), vaga (,Waage'), vagovan/vagovati (,wägen'), zeksar (,Sechser');

- soziales Leben und Lebensbedingungen: ferajn (,Verein'), fremt (,Fremde, Ausland'), oberpav/oberpau (,Oberbau'), farovški (, die Pfarre oder das Pfarrhaus

11 Krevs Birk (2017: 89-91).

12 Weitere Bedeutungen: ,regieren', ,verwalten".

13 Homonym zu štuk, Stukatur'. 
betreffend'), farovž (,Pfarrhaus'), gmajnar (,Bewohner des gemeinschaftlichen Besitzes'), kvartir (,Quartier') ${ }^{14}$, obrajtati (,jdn. schätzen'), šelm (,Schelm'), tajčat (, auf Deutsch reden'), tajfel (,Teufel'), viža (,Art und Weise'), žihar (Partikel in der Bed. ,dürfen'), žlahta (,Verwandtschaft'), kasa (,Kasse'), ohcet (,Hochzeit'), plac (,Platz', auch Name des Ortzentrums von Kropa: Plac), rabutanje/rabutati (,Obst vom Ostgarten klauen'), rajža (,Reise'), ravbšic (,Raubschütze'), revir (,Gebiet'), rotovž (,Rathaus'), šnofati (,schnüffeln'), špilati (,spielen' (Musik)), špital (,Krankenhaus'), štant (,Verkaufsstand', ,Jägerstand'), štelenga (,Stellung, Musterung'), štiftati (, spenden, schenken') ${ }^{6}{ }^{15}$, vahta (,Wacht'), verbati (,erben'), zažingati (,zum Singen anfangen');

- Berufe und Berufe betreffend: fajmošter (,Pfarrer, Pfarrmeister'), firšt (,Fürst'). oberlajtnant (,Oberleutenant'), rihtar (,Richter'), šomošter (,Lehrer'), žandar (,Gendarm'), žnidar (,Schneider');

- Handwerk, insbesondere Schmiedehandwerk: cimaraka (,Zimmerhacke'), četna (,Kette'), dila (,Diele, Brett'), krunkelj (,Krummholz'), kufren (, aus Kupfer'), kugla (,Kugel'), paganet (,Bajonet'), fabrika (,Fabrik'), cajn (,Zain, Stabeisen'), jeger (,Stahlnagelart'), ješa (,Schmiedesse'), kapselj (,Zünder'), ogomošter (,Hammermeister als Vorarbeiter');

- andere: bart (,-mal'), gvišnə (,sicher, gewiss'), retur (,zurück').

\section{5}

Unter den mundartlichen Germanismen finden sich einige wenige Lexeme, die formativisch (phonologisch, morphologisch) vom deutschsprachigen Herkunftswort vollkommen abweichen und dem Slowenischen phonetisch und morphologisch in dem Maße angepasst sind, dass sie die deutschsprachige Herkunft im Gegenwartsslowenisch kaum oder gar nicht erkennen lassen (z. B. firbec < mhd. virwiz, ahd. furewizze, Vorwitz', ješa $<$ mhd. esse, ahd. essa ,Esse'), und solche, die ihren deutschsprachigen Ursprung sehr wohl zu erkennen geben, da die primäre und sekundäre Adaption (S. Stojić/Turk 2017) vor allem graphematisch und morphonologisch (z. B. Genus: fremt $\mathrm{m}<$ Fremde f, kovtar $<$ mhd. kolter f) war, wie etwa ferajn, kontribucion, ajzenponar, kasa, fabrika, fremt, kvartir usw. Dabei handelt es sich um Lexeme, die seit dem späten Mittelalter ins Slowenische übernommen wurden, was auch mit den Sprachkontaktprozessen regionalweit seit der Ansiedlung der deutschen Muttersprachler in Kropa und später (ab dem 13./14 Jh.) zusammenfällt. Nach den etymologischen Festlegungen Striedter-Temps' (1963) und Snojs (1997 und fran.si), ${ }^{16}$ die weitgehend übereinstimmen, stammen die meisten im Kap. 3.4 genannten Lehnwörter aus dem Mhd. sowie aus dem Nhd. Darunter sind auch

14 Manche Wörter wie etwa kvartir kamen aus dem Französischen über das Deutsche ins Slowenische im Rahmen des Soldatenswortschatzes, den Valentin Vodnik in der Zeitung Lubljanske novice durch Medienberichte aus aller Welt verbreitete (Smolej 2018: 27).

15 Historisch auch: ,gründen'.

16 S. Snoj, Marko, Slovenski etimološki slovar ${ }^{3}$, www.fran.si (01.06.2020). S. auch Šekli (2015) und Javor Briški (2020). 
Wörter, die dem Französischen entstammen und durch das österreichische Deutsch vermittelt wurden wie etwa oberlajtnant, šansa, retur, vestja, žandar. ${ }^{17}$

\section{6}

Die slowenischen Dialekte im Gorenjsko-Gebiet waren mit den Kärntner-slowenischen Dialekten im Kontakt, aus Gründen der Koexistenz im selben Gebiet waren diese Kontaktvarietäten auch im Sprachkontakt mit den Chronolekten des südbairischen Oberdeutschen, das sich geographisch mit dem Norden des slowenischen Dialektkontinuums überlappt. Darüber hinaus traten die slowenischen Kontaktvarietäten auch mit weiteren Varietäten des Bairisch-Österreichischen in Kontakt, sowie - in der Neuzei - mit dem (über-)regionalen österreichischen Wortschatz ${ }^{18}$, unter anderem auch mit der Lexik der (Beamten)Sprache der ehemaligen k. u. k. Verwaltung. Unter den untersuchten Germanismen gibt es somit keinesfalls wenige, die aus dem bairisch-österreichischen Dialekt stammen, z. B. štupa (< mhd. stupp ,Pulver'), dila, pogruntati, retur, šajtrga, šentan, ofer, špital, fejst u. a. Hierbei wundert aber auch nicht die Feststellung, dass einige gemeinsame Germanismen auch im Kroatischen zu finden sind, was im Weiteren auch gezeigt wird. Dies ist sicherlich der Tatsache zuzuschreiben, dass insbesondere die lexikalischen Germanismen in den südslawischen Regionen als Folge des intensiven, mehrere Jahrhunderte lang bestehenden Sprachkontaktes vorhanden sind und dass der slowenische und kroatische Sprach- und Kulturraum unter dem deutschsprachigen Einfluss der politischen Herrscher, zuletzt der Österreichisch-Ungarischen Monarchie, war - wenn auch beide Kontakträume im Dualismus in differente Monarchiehälften fielen. Nicht zuletzt gehörten beide Sprachen zum sog. altösterreichischen Kontaktareal, in dem sich ein Sprachbund herausbilden konnte, zu dem Kontaktsprachen mit konvergentem sprachlichem Material innerhalb des österreichischen Verwaltungsgebietes gehören, wie etwa Polnisch, Tschechisch, Slowakisch, Ungarisch, Slowenisch, Kroatisch, Bosnisch, Serbisch und Ukrainisch (Newerkla 2017: 21). ${ }^{19}$

\section{7}

Im Weiteren wird die substandardsprachliche Varietätendistribution der genannten Lexeme im Slowenischen anhand der variationslinguistischen Verweise auf dem Portal fran. $\mathrm{si}^{20}$ dargestellt. Es ist bemerkenswert, dass keinesfalls wenige genannte

17 Die mundartlichen Germanismen aus der Geschichtssammlung Kroparske zgodbe wurden im Rahmen der Magisterarbeit von Domen Krištofelc (2019) gesammelt und chronolektal differenziert.

18 Zur sprachgeographischen Einteilung des österreichischen Wortschatzes s. Pohl (2011/2012: 6672).

19 Vgl. Anm. 2. S. dazu auch Krevs Birk (2019: 167).

20 Vor allem wurden folgende digitalisierte Wörterbücher überprüft: Wörterbuch der slowenischen Literatursprache (Slovar slovenskega knjižnega jezika - SSKJ ${ }^{2}$ ), das Slowenische etymologische Wörterbuch von Marko Snoj (2015) sowie auf www.fran.si veröffentlichte Dialektwörterbücher (für Dialekte aus dem Zadrečka-Tal, Bovec, Črni Vrh und Kostel) - Weiss (2015): Slovar govorov Zadrečke doline med Gornjim Gradom in Nazarjami (A-H), Ivančič Kutin (2015): Slovar bovškega govora, Tominec (2015): Črnovrški dialekt, Gregorič (2015): Kostelski slovar. 
Germanismen trotz ihrer substandardlichen Prägung ins Wörterbuch der slowenischen Schriftsprache „Slovar slovenskega knjižnega jezika“ (SSKJ) eingetragen wurden. Sie werden den folgenden substandardsprachlichen Varietäten zugeordnet: umgangssprachlich (pogovorno), salopp (nižje pogovorno), dialektal (narečno). Einige Germanismen werden als archaisch (starinsko), historisch (zgodovinsko), pejorativ oder vulgär annotiert, die aber in den in der Kropa-Mundart verfassten Dialogen von „Kroparske zgodbe" vom dialektalen Gebrauch zeugen.

Diese Varietätendistribution im SSKJ sieht folgendermaßen aus:

umgangssprachlich cajnati (aus cajn), fabrika, farovški, farovž, fejst, kasa, kvartir (,Bleibe', ,Wohnung'), likof, ohcet, pisker, (po)gruntati, rabutanje/rabutati, šansa, špilati, vaga, vandrovec, viža, žajfa, žlahta;

salopp: kevder (Kropa-Mundart: čevdr), dila, firbec, gvišnə, košta (Kropa-Mundart: kešt); kovter/kolter (Kropa-Munart kovtar), namalati/malati (,malen', Kropa-Mundart; ,aufschreiben'), naštiman/naštimati se, obrajtati, odrajtati, ofer, plac, rabutanje/rabutati, (s) cagati, šajtrga (und dial.), šmir, špital, štant (,Verkaufsstand'), štenga (Kropa-Mundart auch: štenža), štuk, štupa, tavžent, varžet, zažingati, žihar (žiher), žnidar, župa; dialektal: boršt, cimraka;

archaisch: bogati, galant/galanten; krunkelj (Bovški), kvit (Kostelski), ravbšic (andere Dial.); hist. činž, zeksar, žandar;

archaisch: rajža, regirati, rihtar, šentana (para), šomošter/šolmošter, štibala, oberlajtnant (Militär: ,Oberleutenant'), vagovan/vagovati (Pleteršnik), vestija, pejorativ fajmošter oder vulgär: drek;

ohne Markierung: kontribucija (Kropa-Mundart: kontribucjon). Wenige Germanismen, die eine standardsprachliche Doublette aufweisen, sind ohne varietätenspezifische Markierung eingetragen. Einige werden als domänenspezifisch markiert: norec (Metalurgie: ${ }^{21}$,spezielle Art von Hammer'), oberlajtnant (Militär: ,Oberleutenant'), povštertanc (Ethnologie: ,eine Art Volkstanz'), revir (,Gebiet'), rotovž (Realie: ,Rathaus'), štant (Jägersprache: ,Jägerstand').

Dialektismen in diversen Dialektwörterbüchern (unterschiedlich distribuiert in Zadrečki, Bovški, Črnovrški, Kostelski slovar) ${ }^{22}$ und zugleich in der Kropa-Mundart (im Falle der Variante als erstes Formativ angegeben) sind: antret/antwert, četna/ketna, čevdr/kevder, durhcug, ferajn, fertig, finfar, firšt, foršus, fremt (in Kärntner-Dialekten), gloncati/glancat, kufrast, kugla, lampica/lampa, namalati, pant, priftošel, pubəčlpubec/ pubič/poba), rajtanga, štelenga, vahta, verbati. Kontroht und paganet sind mundartliche Varianten von kontrakt und bajonet. In der Verschriftlichung der Kropa-Mundart in „Kroparske zgodbe“ wurden noch weitere Germanismen gefunden, zu denen es keine Angaben auf fran.si gibt, und zwar: gmajnar, jeger, kufren, oberpav/oberpau, ogomošter, retur, šelm, šnufati (SSKJ šnofati), štiftati (,spenden, schenken'), tajčat, tajfel.

Diese Varietätenübersicht ist relevant u. a. unter dem kontaktlinguistischen Aspekt, denn die Germanismen spiegeln als Reflexe des Sprachkontaktes die rege Sprachkontaktund Kulturgeschichte des Kontaktraumes und speziell der Kropa-Gegend wider, denn

21 Norec bedeutet in der ersten Bedeutung ,Narr` und wurde auch in seiner südbairischen lautlichen Ausprägung abgeleitet.

22 S. fran.si (01.09.2020). 
sie zeugt - obwohl diese Lehnwörter nicht standardisiert wurden - von dem intensiven Sprachgebrauch der Germanismen, von ihrer diatopischen, diastratischen und (im Dialektkontinuum) dialektalen Verteilung wie auch Präsenz im slowenischen Dialektkontinuum. Einige der genannten umgangssprachlichen Germanismen sind fester Bestandteil des gesprochenen Slowenischen, und davon sind in der mündlichen überregionalen Alltagsrede gebräuchlich mindestens folgende Lexeme: fabrika, kevder, dila, bogat, drek, ketna, kevder, durhcug, ferajn, fertik, flavzarija, foršsus, gloncat/glancat, galanten, gvišen, kovter, kregan, kvartir, malati/namalati, ofer, ohcet, pant, pisker, plac, (po)gruntati, štenge, štant, špilati, šnofati, šmir, šansa, šajtrga, scagati, rabutanje, poba, pobič, štuk, vaga, žajfa, žihar/žiher, žlahta.

\section{8}

Wie groß die Reichweite und Intensität des deutschsprachigen Einflusses auf seine Kontaktsprachen - wie etwa auf Nachbarsprachen wie das Slowenische, Tschechische, Ungarische - war, kann man, wie erwähnt, auch an den Sprachkontaktprozessen, die zwischen weiteren Nachbarsprachen des südslawischen Raums herrschten, wie etwa zwischen dem Slowenischen und dem Kroatischen, ablesen. In einem geographischen Kontaktareal, in dem Kroatisch eine der Nachbarsprachen des Slowenischen, zugleich aber keine Nachbarsprache des Deutschen war (obwohl es über vier Jahrhunderte im Verwaltungsgebiet der Habsburgermonarchie lag), ${ }^{23}$ sind auch im Kroatischen zahlreiche Germanismen zu vermerken, ${ }^{24}$ und zwar sowohl im Standard als auch in unterschiedlichen Varietäten (Stojić 2020: 5) des Substandards. Es kann des Öfteren beobachtet werden, dass die in südslawischen Sprachen verankerten Lehnwörter deutscher Herkunft oft als südlich der deutschen Sprachgrenze kursierende überregionale Germanismen zu bemerken sind, die einen hohen Grad an Konvergenz aufweisen, wie den Germanismen-Wörterbüchern von Memić (2014), Glovacki-Bernardi (2013) oder Stojić (2020) zu entnehmen ist. Wenn man die hier beleuchteten Germanismen mit den kroatischen Germanismen, die im Wörterbuch Aneta Stojićs „Germanizmi u hrvatskima govorima“" (2020) aus verschiedenen kroatischen Dialekten eingetragen sind, vergleicht, sind folgende (nonstandardsprachliche) Germanismen konvergent: fejst - kroat. fest, fertik - kroat. fertig, gloncati/glancati - kroat. glancat, gmajnar, jeger ,Stahlnagelart' in der Kropa-Mundart und allgemeinslow. Jäger - kroat. jager ,Jäger', košta, kregan/kregati se - kroat. kregat/kregat se, kufren/kufer (,aus Kupfer'/Kupfer) - kroat. kufar (,Kupfer'), kvartir (,Bleibe') - kroat. kvarter (,Wohnung'), lampa, likof (,Fest nach dem Bauabschluss') - kroat. likaf („Trunk, mit dem der Abschluss eines Handels bekräftigt wird“), malati - kroat. malati/majlat, naštimati, oberlajtnant - kroat. oberlajtnand/oberlajtnand, ofer, pant - kroat. bant, gruntati, ravbšic - kroat. raubšic/rapšicar, rihtar - kroat. rihtar/lihtar, šmir - kroat. šmir/čmir, šnofati, špilati, špital - kroat. špetala/špita/špital/špetalj/špitav/špotol, štenga - kroat. štenga/štige (,Stiege'), vahta, verbati - kroat. erbati,

23 Wie Stojić (2020: 4-6) festhält, kamen die Germanismen durch die deutschen Ansiedler, durch Soldaten an der Militärgrenze wie auch durch das Deutsche als offizielle Verwaltungssprache ins Kroatische.

24 Das Wörterbuch der kroatischen Germanismen enthält 5372 Lexeme (Stojić 2020: 7), wovon, und wie die Autorin betont (ebd.: 5) - lediglich ca. 200 Bestandteil der kroatischen Standardsprache sind. 
erbovati, vandrovec - kroat. vandraoc/vandrač/vandrafčok/vandravec/vandrvandrokaš, slow. zekser (,Sechser') - kroat. sekser/zekser (,Kleingeld'), žajfa, žnidar - kroat. žnjidar/žnjider/šnajdar/šnajder, župa - kroat. supa/župa.

Es handelt sich um mindestens einen Viertel der untersuchten Germanismen, die mit den kroatischen Germanismen aus Stojićs Wörterbuch konvergent sind. Ob dies auf das Bestehen einer Art ,Sprachkontaktkontinuum“ für Germanismen (in Anlehnung an „Dialektkontinuum") schließen lässt, wäre weiter zu untersuchen, indem man noch weitere Sprachvarietäten im interkulturellen Kontakt vergleichen würde. ${ }^{25}$ Hierbei wäre das Sprachkontaktkontinuum im Bereich der dialektalen Germanismen mit der Lexik des „altösterreichischen Kontaktareales“ (Newerkla 2017: 21) bzw. dem „spezifisch-österreichischen Wortschatz als Verwaltungs- und Verkehrswortschatz" (Pohl 2011/2012) zu vergleichen, da die genannten konvergenten Germanismen sowohl aus dem Kontaktareal Altösterreich als auch aus dem weiträumigen Bairisch-Österreichischen und sogar aus weiteren, über die dialektalen Grenzen der Großdialekte hinaus verwendeten deutschen Sprachvarietäten kommen.

\section{SCHLUSS}

Mit der Darstellung der Lehnwörter deutscher Herkunft aus der Verschriftlichung der Mundart wurde gezeigt, wie intensiv die Sprachkontakte zwischen den deutschen Varietäten und der Mundart von Kropa einst waren. Diese Mehrsprachigkeit in der Sprachkontaktentwicklung verursachte in Zusammenhang mit der speziellen geographischen Lage und Homogenität der Bewohner eine ausgesprochen eigenartig ausgeprägte Mundart, deren Diversität und damit verbundene Kultur man nachvollziehen und weiter pflegen sollte. Die Germanismen in der Mundart weisen zugleich auch auf Spezifika der historischen Entwicklung von Kropa als einem Mikrokosmos in der Oberkrain hin, der durch die Geschichte hindurch seit der Ansiedlung der deutschen Muttersprachler Träger eines Sprachkontaktgeschehens war, das zum Kontinuum weiträumiger Kontaktprozesse südslawischer Kulturräume mit verschiedenen Varietäten des Deutschen beiträgt.

\section{Literatur}

BERTONCELJ, Joža (2001) Kroparske zgodbe. Kropa: Tomco.

BOGOŽALEC, Toni (2001) „Nehavanje“. Zgodovinske povezave železarske Krope s sorodnimi kraji na avstrijskem Koroškem. Kropa: Tomco.

Duden online. https://www.duden.de/woerterbuch (02.09.2020).

DWDS - Digitales Wörterbuch der deutschen Sprache. Das Wortauskunftssystem zur deutschen Sprache in Geschichte und Gegenwart. Hrsg. v. d. Berlin-Brandenburgischen Akademie der Wissenschaften. https://www.dwds.de/ (06.11.2020).

25 Das Sprachkontaktkontinuum im Bereich der dialektalen Germanismen wäre insofern vom ,altösterreichischen Kontaktareal“ (Newerkla 2017) bzw. „spezifisch-österreichischen Wortschatz als Verwaltungs- und Verkehrswortschatz" (Pohl 2011/12) auszudifferenzieren, als er vor allem die Sprachkontakte mit Herkunftslexemen aus dem weiträumigen Bairisch-Österreichischen nachweist und nicht nur aus dem Kontaktareal Altösterreich. 
FLORJANČIČ, Saša (Hrsg.) (2005) 20. stoletje. Življenje v Kropi in Kamni Gorici, Radovljica: Muzeji radovljiške občine.

GAŠPERŠIČ́, Jože (1956) Vigenjc. Vodnik po zgodovinskih žebljarskih kovačnicah v Kamni gorici, Kolnici, Kropi in Železnikih. Ljubljana: Tehniški muzej Slovenije.

GAŠPERŠIČ́, Jože (2016) Zbrana. dela. Hrsg. von Gašperšič, Rok. Kropa: Samozaložba.

GREGORIČ, Jože (2015) Kostelski slovar. Ljubljana: Inštitut za slovenski jezik Frana Ramovša ZRC SAZU. www.fran.si (30.08.2020).

IVANČIČ KUTIN, Barbara (2015) Slovar bovškega govora. Ljubljana: Inštitut za slovenski jezik Frana Ramovša ZRC SAZU. www.fran.si (30.08.2020).

JAVOR BRIŠKI, Marija (2020) Nemščina v diahroni perspektivi. Ljubljana: Znanstvena založba Filozofske fakultete.

KÖBLER, Gerhard (1995) Deutsches Etymologisches Wörterbuch. http://www.koeblergerhard.de/derwbhin.html) (Zugriffsdatum: 01.09.2018).

KRANZMAYER, Eberhard (1944) Die deutschen Lehnwörter in der slowenischen Volkssprache. Ljubljana.

KREVS BIRK, Uršula (2015) „Deutsch-slowenischer Sprachkontakt im Lichte der Lehnbeziehungen." In: N. Burneva (Hrsg.), Germanistik in Bulgarien. Bd. I. N. Sofia: Nov bulgarski universitet, 127-138.

KREVS BIRK, Uršula (2017) „Zum aktuellen Status der Germanismen im slowenischen Substandard. Purismus und Gebrauchstendenzen." In: H. Phillip/A. Ströbel (Hrsg.), Deutsch in Mittel-, Ost- und Südosteuropa. Geschichtliche Grundlagen und aktuelle Einbettung: Beiträge zur 2. Jahrestagung des Forschungszentrums Deutsch in Mittel-, Ost- und Südosteuropa, Budapest, 1.-3. Oktober 2015. Regensburg: F. Pustet, 85-99.

KREVS BIRK, Uršula (2019) „Zu einigen Aspekten des Deutschen als Kontaktsprache des Slowenischen." Linguistica 59/1, 155-173.

KRIŠTOFELC, Domen (2019) Germanismen in der Mundart von Kropa. Magistrsko delo. Ljubljana: Filozofska fakulteta, Oddelek za germanistiko z nederlandistiko in skandinavistiko.

LOGAR, Tine/Janez RIGLER u. a. (2016) Karta slovenskih narečij z večjimi naselji. Ljubljana: Inštitut za slovenski jezik Frana Ramovša ZRC SAZU/Geografski inštitut Antona Melika ZRC SAZU/Inštitut za antropološke in prostorske študije ZRC SAZU. https://fran.si/204/sla-slovenski-lingvisticni-atlas/datoteke/SLA_Karta-narecij.pdf (01.09.2020).

MEMIĆ, Nedad (2014) Rječnik germanizama in austrijacizama u bosanskome jeziku. Sarajevo: Connectum.

MEMIĆ, Nedad (2019) „Besonderheiten der deutschen Verwaltungssprache im k. u. k. Bosnien-Herzegowina." Linguistica 59/1, 187-195.

MÜLLNER, Alfons (1909) Geschichte des Eisens in Krain, Görz und Istrien: von der Urzeit bis zum Anfange des 19. Jahrhunderts: mit 177 Illustrationen, einem Faksimile und einer Karte im Text. Leipzig: Halm und Goldmann.

NEWERKLA, Stefan Michael (2017) ,Kontaktareale in Mitterleuropa am Beispiel Altösterreich." In: Ch. Mauerer (Hrsg.) Mehrsprachigkeit in Mittel-, Ost- und Südosteuropa. 
Gewachsene historische Vielfalt oder belastendes Erbe der Vergangenheit. Beiträge zur 1. Jahrestagung des Forschungszentrums Deutsch in Mittel-, Ost- und Südosteuropa, Regensburg, 2.-4. Oktober 2014. Regensburg: Verlag Friedrich Pustet, 17-32.

PLETERŠNIK, Maks (1894; 2014) Slovensko-nemški slovar. Ljubljana: Založba ZRC. https://fran.si/136/maks-pletersnik-slovensko-nemski-slovar) (01.08.20209).

POHL, Heinz-Dieter (2011/2012) „Österreichisches Deutsch. Überlegungen zur Diskussion um die deutsche Sprache in Österreich." Klagenfurter Beiträge zur Sprachwissenschaft, 37-38, 63- 124. http://members.chello.at/heinz.pohl/Pohl_OeDt_ KBS.pdf (10.06.2020).

POHL, Heinz-Dieter (2019) „Deutsch-slowenische Wechselbeziehungen in der Sprache der traditionellen Kärntner Küche." Linguistica 59/1, 253-265.

SEEBOLD, Elmar (2011) Kluge. Etymologisches Wörterbuch der deutschen Sprache. 25., durchgesehene und erweiterte Ausgabe. Berlin/Boston: De Gruyter.

Slovar slovenskega knjižnega jezika, druga, dopolnjena in deloma prenovljena izdaja, www.fran.si (01.08.2020).

SMOLEJ, Mojca (2018), „Bonaparte ne chevauchait en personne entre les lignes de tir et les troupes et attisait le courage dans les ccers.« L'influence du Français sur le vocabulaire militaire dans Ljubljanske novice de Valentin Vodnik." Linguistica $58 / 1,23-32$.

SNOJ, Marko (2009) Etimološki slovar slovenskih zemljepisnih imen. Ljubljana: Modrijan: Založba ZRC.

SNOJ, Marko (2015) Slovenski etimološki slovar. Ljubljana: Založba ZRC. https:// fran.si/193/marko-snoj-slovenski-etimoloski-slovar) (01.02.2020).

STOJIĆ, Aneta (2020) Germanizmi u hrvatskim govorima. Zagreb: Alfa.

STOJIĆ, Aneta/Turk, Marija (2017) Deutsch-kroatische Sprachkontakte. Historische Entwicklung und aktuelle Perspektiven auf lexikalischer Ebene. Tübingen: Narr Francke Attempto.

STRIEDTER-TEMPS, Hildegard (1963) Deutsche Lehnwörter im Slovenischen. Wiesbaden: Harrassowitz. http://lwp.ids-mannheim.de/dict/st (01.02.2020).

ŠEKLI, Matej (2015) „Pomenska polja nemških izposojenk v slovenščini.“ Jezikoslovni zapiski. 21, 2. 31-44. http://www.dlib.si/details/URN:NBN:SI:doc-J0BASPI4 (01.09.2019).

ŠEKLI, Matej (2015) „Semantische Felder der deutschen Lehnwörter im Slowenischen." Slavica TerGestina, 144-168. https://www.openstarts.units.it/bitstream/10077/12248/1/06_slavicaTer_2015_Sekli.pdf (01.03.2020).

ŠKOFIC, Jožica (1996) Glasoslovje, oblikoslovje in besedišče govora Krope na Gorenjskem. Doktorska disertacija. Ljubljana.

ŠKOFIC, Jožica (2001) Govorica jih izdaja. Skica govora kovaške Krope. Kropa: Tomco.

ŠKOFIC, Jožica (2001) „Narečno besedje v Kroparskih zgodbah.“ Vigenjc, glasilo Kovaškega muzeja v Kropi. 1, 17-29.

ŠKOFIC, Jožica (2019) Krajevni govor Krope. Ljubljana: Založba ZRC.

ŠMITEK, Janez (1980) Kovaški muzej v Kropi. Kulturni in naravni spomeniki Slovenije, 68. Ljubljana: Zavod SR Slovenije za spomeniško varstvo. Maribor: Obzorja. 
TOMINEC, Ivan (2015) Črnovrški dialekt. Ljubljana: Inštitut za slovenski jezik Frana Ramovša ZRC SAZU. www.fran.si (30.08.2020).

VALVASOR, Janez Vajkard/Erasmus FRANCISCI/Jožef SISENTSCHELLI/Janez Gregor DOLNIČAR/ Pavao VITEZOVIĆ-RITTER (1689) Die Ehre dess Hertzogthums Crain: das ist, Wahre, gründliche, und recht eigendliche Belegen- und Beschaffenheit dieses ... Römisch-Keyserlichen herrlichen Erblandes. I. Band. III. Buch. http://www.dlib.si/?URN=URN:NBN:SI:doc-NQQSKQM6 (30.05.2020).

WEISS, Peter (2003) „Uvod v (slovenski) narečni slovar.“ Jezikoslovni zapiski. Zbornik Inštituta za slovenski jezik Frana Ramovša, 9, 1, 49-61.

WEISS, Peter (2015) Slovar govorov Zadrečke doline med Gornjim Gradom in Nazarjami $(A-H)$. Ljubljana: Inštitut za slovenski jezik Frana Ramovša ZRC SAZU. www.fran.si (20.08.2020).

\section{Zusammenfassung \\ KONTAKT- UND VARIETÄTENLINGUISTISCHE ASPEKTE DES DEUTSCHEN LEHNGUTES IN DER MUNDART VON KROPA}

Vor über sechs hundert Jahren wurden deutsche Muttersprachler vorwiegend aus Kärnten angesichts des sich stark entwickelnden Eisenhüttenwesens in Kropa, einer Ortschaft in der Oberkrain, angesiedelt. Im Beitrag wird der Sprachkontakt in Kropa behandelt wie auch die deutschen Lehnwörter als Spuren des deutsch-slowenischen Sprachkontaktes in der Mundart von Kropa. Die Kontakte zwischen den Bewohnern Kropas und Kärntnern wie auch diversen Händlern, die Eisenprodukte aus Kropa in anderen Regionen verkauften, hinterließen viele Spuren in der Mundart von Kropa. Im Beitrag werden die substandardsprachlichen Germanismen unter mehreren Aspekten wie etwa der semantischen Felder wie auch der Varietätendistribution im Slowenischen dargestellt. Viele konvergente deutsche Lehnwörter wurden auch im Kroatischen anhand Stojićs Wörterbuchs der Germanismen in kroatischen Mundarten gefunden, was einen intensiven slawisch-deutschen Sprachkontakt widerspiegelt, dessen Spuren regionalweit in Südosteuropa und an dessen Rändern zu finden sind.

Schlüsselwörter: Sprachkontakt, deutsche Ansiedler, Oberkrain, Mundart von Kropa, Germanismus

\section{Abstract \\ GERMAN BORROWINGS IN THE DIALECT OF KROPA: LANGUAGE CONTACT FROM A VARIATIONIST PERSPECTIVE}

The article deals with language contact in Kropa, a settlement in Upper Carniola. More than six hundred years ago, German-speaking migrants coming mostly from what is now Austrian Carinthia settled in Kropa where the metalworking industry was 
developing. The contact between the residents of Kropa and the Carinthians as well as the merchants who sold Kropa metal products in and outside the region, influenced the local Slovene dialect. German borrowings are considered as evident traces of the close interaction between the Slovene and German languages. In the paper, we discuss non-standard German loanwords in terms of their semantic fields and their distribution across different varieties of Slovene. Similar German borrowings have also been found in Croatian and are listed in a recently published lexicon of Germanisms in Croatian dialects. The Kropa case is an example of intense Slavic and German language contact, which is found elsewhere in Southeastern Europe.

Keywords: language contact, German settlers, Upper Carniola, Kropa dialect, German borrowings

\section{Povzetek \\ JEZIKOVNOSTIČNI IN ZVRSTNOSTNI VIDIKI NEMŠKIH IZPOSOJENK V GOVORU KROPE}

Pred več kot šesto leti so se nemški govorci, večinoma Korošci, spričo nahajališč železove rude in razvijajoče se železarske obrti naselili na območju Krope. Kasnejše povezave med prebivalci Krope in Koroško kot tudi stiki z različnimi trgovci, ki so trgovali s kroparskimi izdelki v druge, predvsem južnejše regije, so pustili v govoru kraja vidne sledi, kot so denimo nemške izposojenke.

V prispevku so predstavljeni nemško-slovenski jezikovni stiki na območju Krope ter nemške izposojenke kot posledice stikov v kroparskem govoru. Prispevek obravnava neknjižne germanizme iz Krope $\mathrm{z}$ več vidikov, med drugim tudi $\mathrm{z}$ vidika razpršenosti po slovenskih jezikovnih zvrsteh. Mnogo konvergentnih nemških izposojenk je mogoče najti tudi v hrvaških govorih, kar kaže na intenziven južnoslovansko-nemški jezikovni stik, katerega sledi so vidne v jugovzhodni Evropi kot tudi na njenih robovih.

Ključne besede: jezikovni stik, nemški priseljenci, Gorenjska, govor Krope, germanizem 